Jeffrey E. Senasu, Bozana Alexander, Abayomi E. Akintorin

\title{
Dexmedetomidine as Adjunctive Therapy for Delirium Tremens
}

\begin{abstract}
This case report describes a patient with severe alcohol withdrawal complicated by a potentially difficult airway management that was treated by adjunctive dexmedetomidine infusion. Benzodiazepines are the mainstay of conventional therapy, but they may not be optimal choices in some complicated situations. Our patient underwent excision of the cancer on the floor of the mouth which involved radical neck dissection and flap rotation. During the course of intensive care he developed airway obstruction, in part, due to somnolence. While we need a better understanding of how dexmedetomidine works in delirium tremens, its use as an adjunctive agent appears warranted in complex clinical situations like this one.
\end{abstract}

\section{KEY WORDS}

Dexmedetomidine, delirium tremens, airway obstruction.

DOI: $10.7251 / S M D 1202113 S$

A 62-year-old male with a past medical history of hypertension and hepatitis $\mathrm{C}$, had a squamous cell carcinoma on the floor of the mouth. He underwent excision of the lesion which involved radical neck dissection and flap rotation. The patient was taken to the SICU one hour after the end of surgery because he did not meet criteria for extubation in the operating theater. He was transferred to the general wards on POD\#2 in a stable condition. On POD\#3, he was transferred back to continued intensive care due to altered mental status and partial airway obstruction. A fiberoptic airway examination revealed supraglottic edema. While in the SICU, the patient had received glycopyrrolate, racemic epinephrine by nebulizer, and dexamethasone. In addition, prior to his airway examination, he had received haloperidol, morphine, and lorazepam for "agitation." When it was determined that the airway obstruction likely resulted from somnolence, flumazenil was given to reverse the sedative affect of benzodiazepine. The patient woke up and was able to maintain his airway with no further assistance. However, then he began to exhibit the signs and symptoms of severe alcohol withdrawal. At that time, dexmedetomidine drip was introduced with judicious supplementation of lorazepam. After two days patient was transferred back to the medical floor.

\section{Discussion}

Alcohol withdrawal is a common problem in the peri-operative period for certain adult patients. The extreme form of alcohol withdrawal is delirium tremens, which can result in significant morbidity and mortality if not competently managed. Benzodiazepines are the mainstay of conventional therapy, but they may not be optimal choices in some com-
(Scr Med 2012;43:113)
John H. Stroger, Jr. Hospital of Cook County, Chicago, IL, USA

\section{Correspodence}

Bozana Alexander, MD

John H. Stroger, Jr. Hospital of Cook

County, Chicago, Illinois 6o612, USA

Submitted: August 20, 2012

Accepted: October 16, 2012 plicated situations. We present a patient with severe alcohol withdrawal complicated by a potentially difficult airway that was managed by adjunctive dexmedetomidine infusion.

The influence of noradrenergic neurotransmission in alcohol withdrawal is well established (1-3). The sympathetic system overdrive (anxiety, agitation, increased blood pressure, tachycardia, and tremor) during alcohol withdrawal may facilitate progression of withdrawal symptoms. By decreasing norepinephine release alpha-2 adrenergic agonists may aid in reducing alcohol withdrawal symptoms. ${ }^{3}$ In contrast to benzodiazepines, this class of drugs offers no benefit for prevention or treatment of alcohol withdrawal seizures or delirium tremens. In our patient's case, benzodiazepines given in sufficient doses to control all symptoms would have resulted in over sedation with attendant airway obstruction. While we need a better understanding of how dexmedetomidine works in delirium tremens, its use as an adjunctive agent appears warranted in complex clinical situations like this one.

\section{References}

1. Darrouj J, Puri N, Prince E, Lomonaco A, Spevetz A, Gerber DR. Dexmedetomidine infusion as adjunctive therapy to benzodiazepines for acute alcohol withdrawal. Ann Pharmocother 2008;42:1703-5.

2. Rovasalo A, Tohmo H, Aantaa R, Kettunen E, Palojoki. Dexmedetomidine as an adjuvant in the treatment of alcohol withdrawal delirium: a case report. Gen Hosp Psychiatry 2006;28:362-3.

3. Muzyk AJ, Fowler JA, Norwood DK. Role of a2-agonists in the treatment of acute alcohol withdrawal. Ann Pharmacother 2011;45:649-57. 